US WURK LXVIII (2019), p.

\title{
[1454] Ynkoarte nammen yn it Frysk
}

\author{
Willem Visser
}

\section{Summary}

Frisian has four types of hypocoristic names, viz. diminutive names, 'real' hypocoristics, names ending in $-/ i /(-\langle y\rangle)$, and shortened names. The latter are the subject of this article. Shortenend names are argued to be in accordance with the scheme 'shortened name = diminutive name minus diminutive suffix'. This means that there is a strong formal relationship between these two name types, for which ample evidence is put forward. These names derive from diminutive names by omitting the diminutive suffix. The morphological operation of 'suffix subtraction', however, is rare in Frisian morphology. In fact, it seems to be needed for the derivation of shortenend names only, which renders it a somewhat suspicious option. Shortened names occupy a position in between diminutive names, 'real' hypocoristics, and y-names, that is, they share properties with all these types of names (and differ from them as well). They thus have their own place in the Frisian landscape of names.

\section{Ynlieding ${ }^{1}$}

De grûnfoarm fan persoansnammen wurdt wol gauris omfoarme. Dat kin yn alle gefallen op de trije neikommende wizen. Sa kin fan in namme in ferlytsingsnamme foarme wurde, dêr't yn (1) foarbylden fan jûn wurde:

1. Op 24 april 2018 haw ik op de '1st Conference on Frisian Humanities' in lêzing oer dit ûnderwerp holden. De oanwêzigen dêr wol ik hjirby betankje foar de fragen en opmerkingen yn it neipetear; ik haw der myn foardiel mei dwaan kinnen. Siebren Dyk komt in wurd fan tank ta foar it besjen fan de earste ferzje fan dit stik; syn op- en oanmerkingen is it abslút net minder fan wurden.

Yn myn stik oer $y$-nammen (Visser (2016)) haw ik op side 121-128 ynkoarte nammen ek op it aljemint brocht. It tinken deroer is neitiid fierder gongen en it liek my goed ta om der yn in apart stik nochris oer gear.

De term 'hypokorisme' brûk ik hjir as sammelnamme foar ferlytsingsnammen, flaainammen, $y$-nammen en ynkoarte nammen. Mei 'flaainamme' doel ik allinnich op nammen lykas dy yn (2) hjirnei: Poai (neist Froukje), Kanne (neist Jan), Kei (neist Gjerryt) en Kike (neist Grytsje), d.w.s., nammen dy't neffens de grûnfoarm omfoarme binne nei foarmen mei in ûnmarkearre fonologyske struktuer.

Us Wurk 68 (2019), s. 49-69; https://doi.org/10.21827/5c580f12521e7 
US WURK LXVIII (2019), p.

(1) foarbylden fan ferlytsingsnammen

$\begin{array}{ll}\text { grûnfoarm } & \text { ferlytsingsnamme } \\ \text { Age }(\mathrm{m}) & \text { Aachje (f) } \\ \text { Jan }(\mathrm{m}) & \text { Jantsje (f) } \\ \text { Lolle }(\mathrm{m}) & \text { Lolke (m)/Loltsje (f) } \\ \text { Lolke }(\mathrm{m}) & \text { Lolkje (f) } \\ \text { Wobbe (m) } & \text { Wopke (m) }\end{array}$

Ferlytsingsnammen wurde neffens itselde prosedee fan de grûnfoarm fan persoansnammen ôflaat as ferlytsingswurden fan haadwurden. Sjoch fierder Visser (2003).

In namme kinne ek omfoarme wurde ta in flaainamme. Yn (2) steane in pear foarbylden:

(2) foarbylden fan flaainammen

$\begin{array}{ll}\text { grûnfoarm } & \text { flaainamme } \\ \text { Froukje } & \text { Poai } \\ \text { Grytsje } & \text { Kike } \\ \text { Jan } & \text { Kanne } \\ \text { Gjerryt } & \text { Kei }\end{array}$

Flaainammen falle foaral op troch de ûnmarkearre fonologyske struktueren dy't se hawwe. Sa komme yn de foarbylden yn (2) de markearre oanset $j$ (Jan) en de markearre komplekse oansetten fr- (Froukje), gr-(Grytsje) en gj- (Gjerryt) yn Kanne, Poai, Kike en Kei net werom, wylst de (markearre) rûzer $f$ troch de (ûnmarkearre) ploffer $p$ (Froukje $\rightarrow$ Poai) en de (mear markearre) stimhawwende ploffer $g$ troch de (ûnmarkearre) stimleaze wjergader $k$ (Grytsje $\rightarrow$ Kike, Gjerryt $\rightarrow$ Kei) ferfongen binne. Dêrtroch hat Kike twa deselde bylûden, wat yn flaainammen in soad foarkomt. Sjoch fierder Visser (2010).

Dan kin in namme ek in saneamde $y$-namme neist him krije, sjoch (3) foar guon foarbylden:

(3) foarbylden fan y-nammen

grûnfoarm

Aaltsje

Albert y-namme

Aly

Appy 
US WURK LXVIII (2019), p.

$\begin{array}{ll}\text { Aldert } & \text { Ally } \\ \text { Harmke } & \text { Hammy } \\ \text { Oepke } & \text { Oepy } \\ \text { Sibrichje } & \text { Sipy } \\ \text { Ulbe } & \text { Uppy } \\ \text { Wibren } & \text { Wipy }\end{array}$

$y$-nammen binne twawurdliddich en geane op $-y$ út. Sjoch fierder Visser (2016).

De oerienkomsten en ferskillen tusken ferlytsingsnammen, flaainammen, en $y$-nammen wurde yn it skema yn (4) werjûn:

(4) oerienkomsten en ferskillen tusken ferlytsingsnammen, flaainammen, en y-nammen

$$
\text { ferlytsingsnammen } \quad y \text {-nammen flaainammen }
$$

$\begin{array}{llll}\text { man\&frou } & + & + & + \\ \text { 'rekursiviteit' } & + & - & + \\ \text { suffiks } & + & + & - \\ \text { trogee } & + \text { /- } & + & + \\ \text { tal wurdlidden } & \geq 2 & 2 & \leq 2\end{array}$

Ut (4) kin opmakke wurde:

- dat ien en deselde ferlytsingsnamme, flaainamme of $y$-namme foar manlju en froulju brûkt wurde kin (sjoch 'man\&frou');

- dat fan in ferlytsingsnamme in nije ferlytsingsnamme ôflaat wurde kin (dûbele ferlytsing) en fan in flaainamme in nije flaainamme, mar dat in $y$ namme dy mooglikheid net hat (sjoch 'rekursiviteit');

- dat der by it ôflieden fan ferlytsingsnammen en $y$-nammen gebrûk makke wurdt fan in suffiks, mar by it ôflieden fan flaainammen net (sjoch 'suffiks');

- dat $y$-nammen en flaainammen in trogeysk metrysk patroan hawwe, mar ferlytsingsnammen net persee (sjoch 'trogee');

- dat $y$-nammen strikt twawurdliddich binne, mar ferlytsingsnammen en flaainammen net persee (sjoch 'tal wurdlidden').

As fjirde type binne der wat ik hjir as 'ynkoarte nammen' beneame sil. It giet dêrby om nammen dêr't in ferlytsingsnamme neist stiet. Yn (5) wurde der, oardere neffens de ûnderskate ferlytsingssuffiksen, foarbylden fan jûn: ${ }^{2}$

2. De rychjes yn (5) binne fierhinne basearre op dy yn (31) yn Visser (2016:123). 
(5) foarbylden fan ynkoarte nammen

$\begin{array}{ll}\text { a. ferlytsingsnamme op -je } & \text { ynkoarte namme } \\ \text { Aachje } & \text { Aach } \\ \text { Baukje } & \text { Bauk } \\ \text { Fokje } & \text { Fok } \\ \text { Hylkje } & \text { Hylk } \\ \text { Rykje } & \text { Ryk } \\ \text { Swobkje } & \text { Swobk } \\ \text { Teakje } & \text { Teak }\end{array}$

b. ferlytsingsnamme op -tsje ynkoarte namme

Fetsje (Fet+tsje) Fet

Fintsje Fin

Hiltsje Hil

Ibeltsje Ibel

Ytsje (Yt+tsje) Yt

Jeltsje Jel

Metsje (Met+tsje) Met

Tetsje (Tet+tsje) Tet

Tryntsje Tryn

Tsjaltsje Tsjal

c. ferlytsingsnamme op -ke ynkoarte namme

Beitske Beits

Fimke Fim

Gelske Gels

Gepke Gep

Jantke Jant

Japke Jap

Klaske Klas

Liefke Lief

Nynke Nyn

Saapke Saap

Tsjepke Tsjep

Wopke Wop 
Yn dit stik wol ik dizze ynkoarte nammen besjen mei it each op de formele aspekten en (it aard fan) de foarming (§ 2). Dêrneist wol ik ek, koart, wat sizze oer de gebrûksaspekten $(\S 3)$. $^{3}$

\section{De foarming fan ynkoarte nammen}

Yn (5) hjirboppe bin ik derfan útgongen, dat in ynkoarte namme fan in ferlytsingsnamme ôflaat wurdt. Yn dizze paragraaf wol ik dêr arguminten foar bybringe (§. 2.2). Yn $\S 2.3$ jou ik in ôfliedingsskema foar ynkoarte nammen dat de relaasje tusken de grûnfoarm fan de namme en de ynkoarte foarm werjout. Problematyske útkomsten fan ynkoarting komme yn $\S 2.4$ op it aljemint, wylst, as lêste, yn $\S 2.5$ in morfologyske regel formulearre wurdt. Earst wol ik, yn $\S 2.1$, lykwols koart wat sizze oer de âldens fan dit slach namme.

\subsection{De âldens fan ienwurdliddige nammen}

Ienwurdliddige frouljusnammen lykas Ansk, Bauk en Houk hawwe âlde brieven. Schurer (1939:20) seit dêroer: "Uit de eeuwen vóór 1700 zijn ons overgebracht de volgende vrouwennamen, vaak ook in het aparte spellingskleed van die tijden: Fock, Romk, Ansk, Hylck, Frouck, Imck, Popk, Sjouck, Bouk, Foeck, Wyck, Tjamck, Bayck [Bauck, wv], Siuwk, Swobk". ${ }^{4}$

Yn de Aldfryske oarkunden is te sjen, dat in frouljusnamme as Bauke troch weifal fan it einichste lûd - nei alle gedachten sjwa - ta de koarte, ienwurdliddige foarm Bauk wurde koe, wylst in manljusnamme as Bauka troch reduksje fan it einichste folle lûd (<a〉) oergean koe ta Bauke. De namme Bauke moat dan dus, in skoft alteast, foar manlju en froulju te brûken west

3. Der binne pearen nammen lykas Dineke - Dyn, Fenneke - Fen en Tineke - Tyn. Dat liket derop te wizen, dat -eke by nammen as in aparte froulike (ferlytsings)útgong funksjonearret, in opsje dy't yn Visser (2003:279) neamd wurdt. En al hat men by de $y$ nammen net mei ferlytsing te krijen (Visser (2016:96-98)), op grûn fan nammepearen as Betty - Bet, Elly - El, Epy - Eep en Oepy - Oep soe men dochs oannimme kinne dat -y yn $y$-nammen de funksje fan ferlytsingssuffiks hat. Yn rou-advertinsjes sjocht men wol gauris, dat neist nammen as Wibren en Wibrichje de koarte foarm Wyp stiet. Dy soe dan fan de $y$-namme Wipy ôflaat wêze kinne, mar in streekrjochte ôflieding fan de grûnfoarm liket ek mooglik te wêzen. Ik behein my yn dit stik lykwols ta ynkoarte nammen op grûn fan 'wiere' ferlytsingsnammen.

4. Al liket de formulearring dy kant út te wizen, it wol my net oan dat Schurer derop út wol, dat de rige nammen dy't er hjir jout in folsleine list útmakket. Fierderop neamt er it rychje Sjouck, Jei, Ock, Foek, Jel, dêr't er “enz.” op folgje lit. 
hawwe. Yn 'e rin fan 'e Aldfryske tiid waard dit systeem swakker en begûn it gebrûk fan de ûnderskate foarmen trochinoar te rinnen. ${ }^{5}$

Sokke ienwurdliddige frouljusnammen fynt men ek yn wat tradisjoneel as it (iere) Midfrysk oantsjut wurdt, lykas yn de $17^{\text {de }}$-iuwske gearspraak Ansck in Houck en yn Gysbert Japicx syn fers Tjesck-Moars see-aengste. Guon kinne it oant yn ús tiid útholden hawwe. Meastal moat it lykwols sa gongen wêze, dat sokke nammen earst ferlytsing ûndergongen hawwe en dat der fan de sa foarme ferlytsingsnammen wer ynkoarte nammen ôflaat binne. Schurer (1939:21) seit yn dat ferbân:

En het zijn dezelfde namen [de ienwurdliddige frouljusnammen earder yn it sitaat: Fock, Romk, ensfh., wv], die nog heden ten dage de schoolmeisjes, spontaan als ze zijn in de hitte van het spel, - met verwaarloozing van latere, zachtere vormen elkaar toeroepen: "Do moast, Sjouk! 't Is dyn beurt, Hylk! Né Foek, det 's net earlik en do kreauwst (schelmen) ek mei, smoarge Lutsk!"

Latere, zachtere vormen? Ja, want toen na de gouden eeuw vol kracht en energie de meer slappe en zoetsappige 18e eeuw aanbrak, toen vond men die namen zeker te hard, te "bot" voor zachte en berustende vrouwen en alsof het reeds geen verkleinwoorden waren, werd achter de $k$, eigenlijk $k e,{ }^{6}$ een nieuwe lytsmakker geplaatst en werden Frouck en Hylk en Popk tot Froukje, Hylkje en Popkje verheven. (...) Terug naar het land! Ik bedoel naar onze eigen onvervalschte uitgangen. $\mathrm{Er}$ is ook een gezonde reactie evenwel, die terugkeert naar de $16 \mathrm{e}$ en $17 \mathrm{e}$ eeuwsche vrouwennamen: Sjouck, Jei, Ock, Foek, Jel enz.

It liket sa te wêzen, dat Schurer hjoeddeistige ienwurdliddige frouljusnammen as Sjouk, Jei, Ok, Foek en Jel as ynkoartingen fan de ferlytsingsnammen Sjoukje, Jeike, Okje, Foekje en Jeltsje sjocht. De ôflieding fan sokke ienwurdliddige nammen út ferlytsingsnammen moatte lykwols ûnôfhinklike arguminten foar bybrocht wurde. Yn de paragraaf hjirnei sil dêr in smeet op dien wurde.

5. Dizze wysheid tankje ik oan Oebele Vries.

6. Schurer giet der dus fan út, dat Frouk en Hylk ferlytsingsnammen binne. Hoe't er de einichste $-k$ sjocht, as in 'lytsmakker' op himsels of as it oerbliuwsel fan de lytsmakker-ke, dêr lit er him net oer út. 
2.2 Wêrom oft ynkoarte nammen fan ferlytsingsnammen oflaat wurde moatte

Yn it Frysk binne der moai wat net-gearstalde nammen dy't op in sjwa útgeane. ${ }^{7}$ In namme fan dy foarm is yn fierwei de measte gefallen in manljusnamme. ${ }^{8}$ No binne der pearen nammen te foarmjen mei oan 'e iene kant in manljusnamme op in sjwa en oan 'e oare kant in frouljusnamme sûnder sjwa, dêr't yn (6) foarbylden fan jûn wurde:

(6) foarbylden fan pearen fan in manljusnamme op in sjwa en in frouljusnamme dersûnder

manljusnamme op in sjwa frouljusnamme dersûnder

Hille

Hil

Fimme

Fim

Ale

Aal

Op grûn fan sokke nammepearen soe men oannimme kinne, dat fan in manljusnamme op in sjwa in ynkoarte frouljusnamme, sûnder sjwa, ôflaat wurde kin. Sa'n ofwikseling tusken foarmen op in sjwa en foarmen dêrsûnder hat men ek by haadwurden, sjoch de foarbylden yn (7):

(7) foarbylden fan haadwurden mei en sûnder in einichste sjwa

$\begin{array}{ll}\text { blabze } & \text { blabs } \\ \text { dauwe } & \text { dau } \\ \text { doaze } & \text { doas } \\ \text { fluite } & \text { fluit } \\ \text { hekse } & \text { heks } \\ \text { snotte } & \text { snot } \\ \text { spjelde } & \text { spjeld } \\ \text { stjerre } & \text { stjer } \\ \text { wolle } & \text { wol }\end{array}$

Mar wylst it yn (6) om ferskillende soarten nammen giet, t.w. manljus- en frouljusnammen, hat men yn (7) inkel mei foarmfarianten te krijen. ${ }^{9}$ It liket

7. Ferlytsingsnammen geane ek op in sjwa út, mar sjwa is dêr primêr diel fan it ferlytsingssuffiks.

8. In foech parallel by de haadwurden is, dat in net-gearstald haadwurd op in sjwa, mei in pear útsûnderingen, in $d e$-wurd is.

9. De haadwurden yn (7) binne beide de-wurden. Der binne ek gefallen dat it grammatikaal slachte wikselet, lykas by de souwe - it sou en de oarde - it oard. It lidwurd de is keppele oan de foarm op in sjwa. 
dan ek net oannimlik, dat de relaasje tusken de foarmen yn (6) en (7) gelyk is.

It patroan yn (6) liket him ek te oppenearjen yn oare pearen fan manljusen frouljusnammen, sjoch (8) foar foarbylden:

(8) mear foarbylden fan pearen fan manljusnammen op in sjwa neist frouljunammen dersûnder

manljusnamme op in sjwa frouljusnamme dersûnder

$\begin{array}{ll}\text { Auke } & \text { Auk } \\ \text { Bauke } & \text { Bauk } \\ \text { Feike } & \text { Feik } \\ \text { Houke } & \text { Houk } \\ \text { Ypke } & \text { Ypk } \\ \text { Sjouke } & \text { Sjouk }\end{array}$

Der is lykwols in ferskil tusken de manljusnammen yn (6) en dy yn (8). Yn (6) binne it net-gearstalde nammen, yn (8) binne it ferlytsingsnammen (allegearre mei it suffiks -ke). ${ }^{10}$ As de frouljusnammen yn it rjochter fan de manljusnammen yn it lofter rychje ôflaat wurde, dan giet it hjir om it delearjen fan in part fan it suffiks. Dy foarm fan sjwa-deleesje is allinnich fonologysk te begripen - yn it gefal dat sokke nammen har yn flugge, ferbûne spraak oppenearje - mar op morfologyske grûnen is er net te motivearjen. Deleesje fan in part fan in suffiks is in net-reguliere morfologyske operaasje. It is net oannimlik dat it skrassen fan it segmint sjwa hjir de oergong fan in manljusnei in frouljusnamme bewurkmasterje kin, wylst der oan dy operaasje oars it tafoegjen fan in hiel suffiks te pas komme moat.

Men kin lykwols ta in generalisearjende offlieding fan de ienwurdliddige nammen yn (6), Hil ensfh., en (8), Auk ensfh., slagje mei oan te nimmen dat in ynkoarte namme fan in ferlytsingsnamme offlaat wurdt. Dan komt it der mei de nammen yn (6) en (8) út te sjen as yn (9):

10. Fan in manljusnamme kin mei it taheakjen fan in ferlytsingsútgong in oare manljusnamme of in frouljusnamme offlaat wurde (sjoch Visser (2003)). Yn (8) hat men manlike ferlytsingsnammen.

Histoarysk sjoen binne Auke, Bauke, Feike, Houke, Ypke en Sjouke ôflaat fan Auwe, Bauwe, Feie, Houwe, Ipe en Sjou, nammen dy't net allegear mear yn algemien gebrûk lykje te wêzen. 
US WURK LXVIII (2019), p.

(9) hypoteze oer de ôflieding fan de ienwurdliddige nammen yn (6) en (8)

ferlytsingsnamme

ynkoarte namme

Aaltsje

Aal

Aukje

Auk

Baukje

Bauk

Feikje

Feik

Fimke

Fim

Hiltsje

Hil

Houkje

Houk

Ypkje

Ypk

Sjoukje

Sjouk

Der binne ûnderskate oanwizingen dat ynkoarte nammen in nauwe bân mei ferlytsingsnammen hawwe, mei oare wurden, dat (9) in oannimlike hypoteze oer (de rjochting fan) de ôflieding fan ynkoarte nammen út liket te drukken.

Yn it earste plak giet ferlytsing faak lykop mei brekking en lûdferkoarting. No foarmje ienwurdliddige wurden net de geunstichste kontekst foar dy prosessen. Brekking wol yn sokke wurden noch it bêste, wannear't se op in bylûdkloft útgeane, al binne der ek ienwurdliddige wurden op in inkel bylûd mei brekking, lykas skjin, skuon, ljip en guod (sjoch Visser (2002: 204-205 (§ 4.1.2)). Ienwurdliddige frouljusnammen op in inkel bylûd komme ek mei in brutsen of ferkoarte lûd foar. Dat lit him goed ferklearje mei de oannimming dat in ynkoarte namme fan in ferlytsingsnamme offlaat wurdt en dêr it brutsen of koarte lûd fan oernimt. Dat hat pearen nammen opsmiten mei oan 'e iene kant ienwurdliddige manljusnammen mei in sintralisearjend twilûd/lang ienlûd en oan 'e oare kant ienwurdliddige frouljusnammen mei it 'korresponderjende' brutsen twilûd/koarte ienlûd. Yn (10) wurde de gefallen fan dy ôfwikseling jûn dy't Hoekema (1986) neamt: ${ }^{11}$

11. Hoekema neamt ek it nammepear Sjoerd [sjuət] - Sjurd [sjøt]. Wurden mei de kombinaasje -joe- (-[juə]-) hawwe syngroan faak offlate wjergaders mei -ju- (-[jø]-), lykas djoer - djurder/djurst/djurte en fjoer-fjurje/fjurke/fjurren/fjurrich. Foarmen as djurder en fjurje soenen út 'e brutsen foarmen djworder (út djoerder) en fjworje (út fjoerje) ôflaat wurde kinne, mar -ju- koe ek ûntstien wêze út in al yn 'e Aldfryske tiid ferkoarte lang /o:/-lûd, dat letter nei in posysje foarliker yn 'e mûle opskood is (Visser (2002: 225)). Dêrom is it nammepear Sjoerd - Sjurd net yn (10a) en (11a) opnommen. 
US WURK LXVIII (2019), p.

(10) spoaren fan brekking en lûdferkoarting yn ienwurdliddige frouljusnammen

a. brekking

manljusnamme mei in frouljusnamme mei in sintralisearjend twilûd brutsen twilûd

Nies [niəs] Njis [njıs]

Riem [riom] Rjim [rjim]

Ruerd [ryət] $\quad$ Rjurd [rjøt]

Doed [duət] Duod [dwot]

Oeds [uəts] Uods [vwots]

Roel [ruol] Ruol [rwol]

Beart [bıt] Bjert [bjet]

Geart [giət] Gjert [gjet]

a. lûdferkoarting

manljusnamme mei in frouljusnamme mei in

lang ienlûd

koart ienlûd

Jaap [ja:p] Jap [jap]

Klaas [kla:s] Klas [klas]

It brutsen en it koarte lûd yn de nammen yn de rjochter rychjes kin men op ienfâldige wize ferklearje troch oan te nimmen dat dy nammen ynkoartingen fan in ferlytsingsnamme binne, sa't yn (11) oanjûn wurdt:

(11) ynkoarting by de nammen yn (10)

a. $\quad$ nammen mei brekking

$\mathrm{Njis}$

Rjim

Rjurd

Duod

Uods

Ruol

Bjert

Gjert

b. nammen mei lûdferkoarting

Jap

Klas
Njiske

Rjimke

Rjurdsje

Duodsje

Uodske

Ruoltsje

Bjertsje

Gjertsje

Japke

Klaske 
It (folle) lûd fan Klaske en Klas is /a/, wylst, foar it tosklûd /s/, it healiepene efterlûd / $/$ ferwachte wurde mocht. As /a/ in ferkoarting fan in lange /a:/ is, dan kin er foar in tosklûd stean, sa't men dat hat yn bygelyks hastich, hazze en master. It koarte /a/-lûd fan Klaske wjerspegelet dus direkt de 'derivasjonale skiednis' fan dy namme út Klaas en yndirekt dy fan Klas út Klaske. Itselde soe men bygelyks hawwe kinne mei At /at/, in ferkoarting fan Atsje /atsjə/, op syn bar ôflaat fan Ate /a:tə/; en al bin ik de foarm At net tsjinkommen, hy liket my sûnder mear mooglik te wêzen (sjoch ek nulhypoteze (13) hjirnei).

Yn it twadde plak binne der ynkoarte nammen op - $\{p / b\} k$, lykas $Y p k$, Wypk en Swobk. De bylûden /p/ en /b/ binne de iennichste ploffers dy't it ferlytsingssuffiks -ke selektearje (Ype+ke $\rightarrow$ Ypke, Wibe+ke $\rightarrow$ Wypke, Swobbe $+k e \rightarrow$ Swobke). In ferlytsingsnamme op -p/b+ke kin op 'en nij ferlytse wurde, mar no, nei $-k$, mei it suffiks -je, wat altyd in frouljusnamme opsmyt: Ypke+je $\rightarrow$ Ypkje, Wypke+je $\rightarrow$ Wypkje, Swobke+je $\rightarrow$ Swobkje (sjoch Visser (2003:295-296)). By it ynkoartsjen fan sa'n dûbel ferlytse namme komt it op in foarm mei de 'net-kanonike' einkloft -/pk/ of -/bk/ út. Yn net-gearstalde wurden komme dy net foar, wat in oanwizing is foar it offlate karakter fan nammen lykas Ypk, Wypk en Swobk. ${ }^{12}$ De ienfâldichste ferklearring foar sokke einkloften hat men mei de oannimming dat dy nammen ynkoartingen fan in ferlytsingsnamme binne.

Der binne, yn it tredde plak, mear froulike as manlike ferlytsingsnammen (Visser (2003)). Dat giet lykop mei it feit dat der mear froulike as manlike ynkoarte nammen binne. Mei de oannimming dat in ynkoarte namme fan in ferlytsingsnamme offlaat wurdt, hat men in ferklearring foar dizze parallel. ${ }^{13}$

En ta beslút, de einichste sjwa fan in haadwurd en ek fan de grûnfoarm fan in persoansnamme wurdt systematysk skrast foar it ferlytsingssuffiks. Yn ferlytsingsnammen fynt men dy sjwa dus net werom, wat ek jildt foar de ynkoarte nammen dy't dermei korrespondearje.

Der binne, alles mei-inoar, dus de nedige oanwizingen dat in ynkoarte namme fan in ferlytsingsnamme ôflaat wurdt.

12. Sokke ûngewoane einkloften steane ûnder fonologyske druk, dat it hoecht gjin nij te dwaan, dat se bleat lizze foar ferienfâldiging. Sa fernijt Siebren Dyk my, dat syn earder buorfanke Wypkje dei en wei Wyp neamd waard, en net Wypk.

13. In ynkoarte namme komt jin yn 'e gewoane regel as in frouljusnamme oan. Men soe ditoangeande oan in eksperimint tinke kinne. Nim de ferlytsingsnammen Gepke en Simke, dy't foar froulju en manlju brûkt wurde kinne. Wêr tinkt in Fryskprater by de ynkoarte nammen Gep en Sim no as earste oan, in frou- of in manminske? Jins yntuysje seit: in frouminske. Dêr kin fansels in frekwinsje-effekt mei ûnder spylje. 


\subsection{In ofliedingsskema foar ynkoarte nammen}

De formele relaasje dy't in ynkoarte namme mei in ferlytsingsnamme hat, wurdt mei it skema yn (12) útdrukt:

\section{ofliedingsskema foar ynkoarte nammen}

ynkoarte namme = ferlytsingsnamme sûnder ferlytsingssuffiks

Dit skema is algemien formulearre, sûnder ferwizing nei spesifike klassen fan nammen en spesifike ferlytsingssuffiksen. Dat slút oan by wat neffens my as de nul-hypoteze oangeande (it foarmjen fan) ynkoarte nammen jilde moat:

\section{nul-hypoteze oangeande (it foarmjen fan) ynkoarte nammen}

elke ferlytsingsnamme kin in ynkoarte wjergader hawwe

No is (13) te rom formulearre, want men komt dêrmei op tefolle ynkoarte nammen út, oars sein, der wurdt oergenerearre. Der liket nammentlik in rem op dit slach nammefoarming te wêzen, útdrukt troch de outputkondysje yn (14):

$$
\text { outputkondysje op (13) }
$$

de ynkoarte namme moat leafst net gelyk wêze oan in al besteande namme

Neffens dizze kondysje binne ynkoarte nammen as Foek (<Foekje), Jel (< Jeltsje), Rins $(<$ Rinske) en Ibel $(<$ Ibeltsje) sûnder mear besteanber en akseptabel, wylst Jan (< Jantsje), Wierd (< Wierdsje) en Hessel (< Hesseltsje) der as ynkoartingen net op troch kinne of yn alle gefallen lang sa oannimlik net binne. ${ }^{14}$

14. Sa komt bygelyks de ynkoarte frouljusnamme Pyt, fan Pytsje, wol foar, wylst de manljusnamme $P y t$ ek al bestiet. Neist de frouljusnamme Piterke hat men lykwols net de ynkoarte namme Piter. Net allinnich is Piter in al besteande namme, it is ek nochris sa dat in namme op -er altyd in manljusnamme is, wylst by ynkoarting in frouljusnamme in frouljusnamme en in manljusnamme in manljusnamme bliuwt. Men hat hjir dus in strukturele beheining op it foarmjen fan ynkoarte nammen te pakken

De ynkoarte foarm kin ek sa oanpast wurde, dat er der as in 'kanonike' ynkoarte namme op troch kin. Ut in rou-advertinsje wie op te meitsjen, dat Hesseltsje wol Hes neamd waard, nei alle gedachten om betizing mei de besteande manljusnamme Hessel, de reguliere ynkoarting fan Hesseltsje, foar te wêzen. 
Wat it effekt fan (14) oangiet, is der in ferskil tusken grûnfoarmen dy't op in sjwa útgeane en grûnfoarmen dêr't dat net foar jildt. Nim, as earste, nammen dy't net op in sjwa útgeane en de dêrfan ôflate ferlytsingsnamme, bygelyks Jan - Jantsje en Sjoerd - Sjoerdsje. Mei Jantsje en Sjoerdsje komt it neffens skema (12) mei de ynkoarte namme wer op it útgongspunt, de grûnfoarm, út, hjir Jan en Sjoerd. Soks koe maklik foar betizing soargje, omdat Jan en Sjoerd dan in manljus- likegoed as in frouljusnamme wêze soenen, oars sein, it soenen nammen wêze dy't neffens de foarm op dit punt net faninoar te ûnderskieden binne. ${ }^{15}$ Komt it mei it ynkoartsjen fan in ferlytsingsnamme wer op it útgongspunt - de grûnfoarm fan de namme - út, dan feroaret der by einsluten neat en hat sa'n operaasje gjin funksje. De oannimming dêrby is, dat in morfologyske operaasje noait 'samar' of 'om 'e nocht' syn beslach krijt en altyd in beskate funksje hat, oars sein, dat der altyd in beskaat grammatikaal, pragmatysk of stilistysk effekt mei anneks is.

Mei nammen op in sjwa leit dit oars. De einichste sjwa fan de grûnfoarm fan de namme falt wei by it foarmjen fan in ferlytsingsnamme (krekt sa't men dat by ferlytsingswurdfoarming fan haadwurden hat). Yn sa'n gefal komt it neffens skema (12) by ynkoarting op in foarm út dy't ien wurdlid minder hat as de grûnfoarm, en dêrmei ferskille ynkoarte namme en grûnfoarm faninoar. Men krijt sa de trijeslach grûnfoarm - ferlytsingsnamme ynkoarte namme, bygelyks Hille - Hiltsje - Hil en Jouke - Joukje - Jouk. Elk fan dy trije nammen hat syn eigen foarm en funksje. Dat is neffens it One Form One Meaning-Principle, dat seit dat der yn taal in (sterke) oanstriid is ta in ien op ien-relaasje tusken foarm en betsjutting, oars sein, dat de taalbrûker foarmen dy't faninoar ferskille graach oan in ferskil yn betsjutting of funksje keppelje wol (en dus leaver net oan ien en deselde betsjutting of funksje). ${ }^{16}$ It foarmferskil is krusiaal, want dêrsûnder kin men net witte oft men mei de grûnfoarm fan de namme of de ynkoarte ferlytsingsnamme te krijen hat. ${ }^{17}$

15. Sokke ynkoarte foarmen kinne wol gewoan oflaat wurde, mar se binne yn 'e praktyk fan it nammeferkear net handich en nuttich.

16. It giet hjir, nettsjinsteande de namme, dus mear om in (sterke) tendins as om in (absolút) prinsipe.

17. Men kin ek op in oare wize fan in grûnfoarm mei mear as ien wurdlid op in ienwurdliddige namme útkomme, by flaainammen bygelyks (sjoch Visser (2010)). In ynkoarte namme stiet lykwols systematysk in ferlytsingsnamme neist, en it is de ferlytsingsútgong dy't systematysk weifalt. Ferlykje yn dat ferbân bygelyks Wys, dêr't Wysje neist stiet, mei Lys, de flaainamme fan Lysbeth (dat wer fan Elisabeth komt). 


\section{4 problematyske útkomsten fan ynkoarting}

In wurd, en in namme dus ek, moat der fonologysk besjoen op troch kinne. By ynkoarte nammen hat dat der bytiden wol oan.

Krekt as by haadwurden spilet it einichste bylûd fan de grûnfoarm fan in namme in beskiedende rol by de seleksje fan it ferlytsingssuffiks. Yn 'e gewoane regel geane ynkoarte nammen út op it bylûd dat dy seleksje beskiedt. Meidat it foar dy seleksje neat útmakket, oft dat bylûd op himsels stiet of diel is fan in kloft, kinne ynkoarte nammen ek op in bylûdkloft útgean, lykas mei Gelk (neist Gelkje), Gels (neist Gelske) en Syts (neist Sytske). Oan 'e oare kant kinne se ek mei in bylûdkloft begjinne, lykas mei Tryn (neist Tryntsje), Swobk (neist Swobkje) en Tsjep (neist Tsjepke). Ynkoarte nammen hawwe dus net de ûnmarkearre fonologyske struktuer dy't sa karakteristyk is foar flaainammen (in skaaimerk dêr't yn de ynlieding op wiisd is). Dy mear markearre foarm slút oan by de ferlytsingsnammen.

It Frysk hat in soad (frouljus)nammen op -kje, mei histoarysk sjoen dûbele ferlytsing, lykas Lolkje (Lolle+ke+je) en Rinskje (Rinse+ke+je) (Visser (2003:295-296)). Syngroan sjoen giet it hjir lykwols om inkele ferlytsing, dat neffens skema (12) falt by it offlieden fan de ynkoarte foarm fan sokke nammen it suffiks -je derby wei. ${ }^{18} \mathrm{De}-/ \mathrm{k} /$, dy't de seleksje fan -je beskiedt, kin dan foar in minder gebrûklik rym fan it wurdlid soargje, ${ }^{19}$ dat yn (15) yllustrearre wurdt.

(15) fan nammen op -kje ôflate ynkoarte foarmen mei in problematysk rym
a. Aafkje
Aafk
Etkje
Etk
b. Ymkje
Ymk
c. Earkje
Eark
Eelkje
Eelk

- Yn (15a) - Aafk en Etk - giet it om einkloften fan obstruinten dy't men yn net-gearstalde wurden net hat. Yn ôflate foarmen, lykas hifkje, komt -fk- wol foar. In nominalisearring as gehifk (Kinne wy no ek ris ophâlde mei dit

18. Haadwurden op - $/ \mathrm{y}(\partial) /$ wurde ferlytse mei it suffiks -je. Tusken de stam en -je wurdt in /k/ ynfoege, dat by einsluten komt it út op in foarm op -kje, lykas yn dinkje (neist ding) en tankje (neist tange). De sekwinsje -kje yn dûbel ferlytse nammen is lykwols net bûn oan in foarôfgeande $/ \mathrm{y} /$ en kin dan ek net lykslein wurde mei de útgong -kje fan ferlytsingswurden.

19. It rym fan it wurdlid is it rjochter diel, rekkene fan it lûd ôf. Yn Eark en Eelk falle wurd, wurdlid en rym fan it wurdlid dus mei-inoar gear. 
gehifk en in beslút nimme?) is ynoarder. Yn $\S 2.2$ hjirfoar hawwe wy al sjoen, dat nammen op - $\{p / b\} k$, lykas Wypk en Swobk, foarkomme, wylst der gjin net-gearstalde wurden op - $\{p / b\} k$ binne.

- Yn (15b) - Ymk - hat men de net-homorgane kloft fan it twalippige noaslûd $/ \mathrm{m} /$ en de kielploffer $/ \mathrm{k} /$, wylst de kombinaasje fan in noaslûd en in ploffer oan 'e ein fan in stam homorgaan wêze moat. ${ }^{20}$ De kloft $-/ \mathrm{mk} /$ hat men ek yn it bywurd jamk, dat in ôflate foarm, evenlik, as histoaryske basis hat (Hoekstra (1998b)). Sa kin in offwikende, net-kanonike wurdein de derivasjonele skiednis fan it wurd yn kwestje 'ferriede'.

- Yn (15c) - Eark en Eelk - sit it probleem hjiryn, dat de kombinaasje fan de lange fokalyske sekwinsje ealee mei de bylûdkloft -rk/-lk in te lang rym fan it wurdlid opsmyt. Yn 'e gewoane regel komme mar allinnich de tosklûden /d,t,s,z/ oan 'e ein fan sa'n (te lang) rym fan fjouwer posysjes foar, sa't men dat hat yn bygelyks deugd, naald, mient, krêft, en least. /k/ is lykwols in kiellûd. Wurden op $-/\{r / l\} k /$ binne hiel gewoan, mar dan mei in koart lûd, lykas yn ark, merk, bjirk, wurk, kalk, elk, swilk en folk.

Mar al hawwe de ynkoarte nammen hjirboppe allegear in minder gebrûklike foarm en binne se dêrtroch fêst ek minder gewoan, se komme likegoed foar. $^{21}$

\section{5 de formele ferantwurding fan de ynkoarting}

It skema yn (12) seit: ynkoarte namme = ferlytsingsnamme sûnder ferlytsingssuffiks. Om fan in ferlytsingsnamme op in ynkoarte namme te kommen, moat it ferlytsingssuffiks dus skrast wurde. ${ }^{22}$ Dêrmei liket it foarmjen fan ynkoarte nammen in gefal fan 'suffikssubtraksje' te wêzen. Dat morfologysk prosedee komt yn it Frysk oars net foar. Yn 'e Fryske morfology hat

20. Men hat ek de ynkoarting $Y m$, dêr't dit probleem him fansels net foardocht.

21. Neist Nynke stiet Nyn /nin/, mei de ûnderlizzende einichste /n/, sa't ek ferwachte wurde mocht, omdat de /y/ yn Nynke kontekstueel, troch de /k/ dernei, beskaat wurdt. Sa steane neist bygelyks Janke en Minke de $y$-nammen Janny en Minny, mei de $/ \mathrm{n} / \mathrm{fan}$ Jan en Minne, en net mei in /y/. Mar Jan /jan/ en Min /min/ kinne der as de ynkoarte nammen fan Janke en Minke by my net op troch. By Jan soe men dat hirop smite kinne, dat de /a/, foar it tosklûd /n/, op in fonotaktysk besjoen ferbean plak stiet, al hat /a/ yn frouljusnammen in apart plak, tink oan Anne, dat as manljusnamme as [ə:nə/oənə] en as frouljusnamme as [anə] útsprutsen wurdt (sjoch Visser (2016:116))). De foarm /jon/ is fonotaktysk sjoen yn 'e es, mar as ynkoarte foarm fan Janke soe dy fansels maklik foar betizing mei de manljusnamme Jan soargje kinne. Sokke beswieren binne der net mei Min, mar dy foarm is by my like ûnmooglik as Jan. In ferklearring haw ik dêr net foar.

22. Neffens Hoekema (1986:55) kinne frouljusnammen "it suffiks falle litte". 
men wol 'trunkaasje', mar dêrby giet it om morfemen dy't a) gjin betsjutting hawwe (saneamde augminten, helpstikjes) en b) allinnich skrast wurde wannear't der in oar morfeen oan it wurd, en oan deselde kant derfan, taheakke wurdt. Sjoch (16) foar in pear foarbylden, allegearre út Hoekstra (1998a):

(16) foarbylden fan trunkaasje

$\begin{array}{ll}\text { grûnfoarm } & \text { ôflate foarm mei trunkaasje } \\ \text { Drachten } & \text { Drachten+ster } \\ \text { sunich } & \text { súnim+tsjes } \\ \text { gebrûk } & \text { (yn) ûnebrûk } \\ \text { ferbrizelje } & \text { tefexbrizelje }\end{array}$

Yn 'e gewoane regel hat oflieding yn it Frysk lykwols de foarm fan affigearring, d.w.s. it tafoegjen fan in affiks oan in stam. In affiks fertsjintwurdiget beskate 'ynformaasje'. In affigearre foarm hat dan ek mear ynformaasje yn as de foarm dêr't de affigearring op ynwurket. Nim it taheakjen fan it suffiks -ing oan in tiidwurdstam (lykas lês - lêzing) of -ich oan in haadwurd (lykas stien - stiennich). It suffiks -ing fertsjintwurdiget hjir primêr de ynformaasje 'nominalisearring' en -ich 'adjektivisearring', dy't de stammen lês en stien net ynhawwe. Lit men it affiks wei, dan wurdt dy 'ekstra ynformaasje' ek wei en komt it wer op de tiidwurdstam en it net-ôflate eigenskipswurd út.

Fan in namme kin in ferlytsingsnamme foarme wurde. In gebrûklike ynstansjaasje fan 'mear ynformaasje' is hjir, dat de ferlytsingsnamme it skaaimerk <froulik> hat, wylst de grûnfoarm fan de namme dat net hat, bygelyks by de pearen Ruerd <manlik> - Ruerdsje <froulik> en Wytse <manlik> - Wytske <froulik>. By it ynkoartsjen fan in ferlytsingsnamme wurdt it suffiks weilitten. De 'ekstra ynformaasje' dy't it ferlytsingssuffiks fertsjintwurdiget, bliuwt lykwols beholden: in ynkoarte frouljusnamme bliuwt in frouljusnamme. ${ }^{23}$ Dat jildt ek foar de morfonologyske effekten dy meitsje ek ynformaasje út - dy't ferlytsing op de grûnfoarm fan de namme hat of hawwe kin, t.w. sjwa-deleesje, brekking en lûdferkoarting. Mei it weifallen fan it affiks wurdt de 'ekstra ynformaasje' net wei, oars sein, it

23. Al binne der gâns mear froulike as manlike ferlytsingsnammen, dy lêsten komme wol foar, lykas Tsjepke en Wopke (Visser (2003)). De ynkoarte foarmen dêrfan binne de manljusnammen Tsjep en Wop. Sa't in ynkoarte frouljusnamme in frouljusnamme bliuwt, sa bliuwt in ynkoarte manljusnamme dus in manljusnamme. 
weifallen fan it affiks liedt net ta de folsleine 'restauraasje' fan de grûnfoarm fan de namme.

Neffens de outputkondysje yn (14) moat de ynkoarte namme leafst net gelyk wêze oan in al besteande namme, oars sein, der moat in foarmferskil wêze tusken de ynkoarte ferlytsingsnamme en de grûnfoarm fan de namme dêr't de ferlytsingsnamme fan ôflaat is. ${ }^{24}$ Yn $\S 2.3$ haw ik yn dit ferbân it One Form One Meaning-Principle yn stelling brocht: der is yn taal in (sterke) oanstriid ta in ien op ien-relaasje tusken foarm en betsjutting, oars sein, de taalbrûker wol foarmen dy't faninoar ferskille graach oan in ferskil yn betsjutting of funksje keppelje.

No is homonymy - it foarkommen fan neffens de foarm gelikense, mar neffens de betsjutting ûngelikense wurden - in tige algemien ferskynsel (besjoch mar ris in pear siden yn likefolle watfoar wurdboek). Nammen lykje dêr lykwols in tsjinnichheid yn te hawwen. Dat hat fêst te meitsjen mei in ferskil yn funksje tusken nammen en 'gewoane wurden'. De lêsten hawwe in leksikaal-konseptuele betsjutting en der wurde konsepten mei oantsjut, wylst de earsten earst en meast in identifisearjende funksje hawwe. Foar dat lêste is in ien op ien-relaasje tusken foarm en funksje it bêste. Dêrby liket it net ûnlogysk dat it ien fan de wichtichste identifisearjende funksjes fan nammen is om manlju en froulju útinoar te hâlden, yn oansluting by in fûneminteel ûnderskied binnen de minsklike soart.

Dat wol net sizze, dat in namme yn begjinsel noait foar manlju én froulju brûkt wurde kin. By ferlytsingsnammen, flaainammen en $y$-nammen komt dat foar, by de lêste twa typen sels in soad. Dat binne lykwols allegear ôflate nammen, dy't yn in beskate relaasje steane ta oare nammen, dy't yn 'e gewoane regel foar manlju óf froulju brûkt wurde. Datoangeande steane grûnfoarmen mear op harsels. It leit yn 'e reden, dat men dêrmei primêr nei manlju óf froulju ferwize kinne wol.

It ferskil tusken in ferlytsingsnamme en de dêrmei korrespondearjende ynkoarte namme is suver formeel fan aard: de oanwêzichheid (ferlytsingsnamme) vs. de ôfwêzichheid (ynkoarte namme) fan it ferlytsingssuffiks. In formele morfologyske regel foar de foarming fan ynkoarte nammen wurdt yn (17) jûn:

regel foar it foarmjen fan ynkoarte nammen

$$
[\mathrm{X}]_{\text {namme }}+\mathrm{DIM} \rightarrow \quad[\mathrm{X}]_{\text {namme }}
$$

24. Sa't oan de pearen nammen yn (10) te sjen is, kin dat foarmferskil ek suver fonologysk fan aard wêze. 
Sa't hjirfoar opmurken is, hat offlieding yn it Frysk yn 'e gewoane regel de foarm fan affigearring, it tafoegjen fan in affiks oan in stam. Suffikssubtraksje is net in regulier morfologysk prosedee. It liket oars wol foar te kommen. Sa is yn it Nederlânsk op basis fan it ferlytsingswurd toetje $($ toe + tje $)$ it wurd toet foarme ${ }^{25}$. It opfallende is hjir a) dat der in werstrukturearring west hawwe moat (toe + tje $\rightarrow$ toet + je) en $b$ ) dat it wurdslachte feroaret: het toetje wurdt de toet, sa't ek bliken docht oan de bûging fan it eigenskipswurd: een lekkere toet vs. een lekker toetje. Yn it Frysk haw ik wol heard dat fan it ferlytsingswurd suertsje 'slop, ûnwis persoan; immen dy't net doar, neat weaget' de fariant suert brûkt wurdt (Wat in suert!, Dat is wol sa'n suert!). Oft it wurdslachte dan ek feroaret - it suertsje wurdt de suert - doar ik net sizze. Unoannimlik liket soks oars net te wêzen, foaral ek omdat mei suert in persoan oantsjut wurdt en oantsjuttingen fan libbene wêzens meast de as lidwurd hawwe.

No binne toet en suert losse, ynsidintele gefallen. Systematyske suffikssubtraksje wurdt allinnich brûkt foar it ôflieden fan ynkoarte nammen. Mar nammen hawwe wol mear bysûndere morfologyske eigenskippen neffens de oare wurdsoarten, sa't de Dútske nammekundige Damaris Nübling yn ûnderskate publikaasjes sjen litten hat (lykas yn Nübling (2017)).

\section{Gebrûksaspekten fan ynkoarte nammen}

By it brûken fan ferlytsingsnammen, flaainammen en $y$-nammen - by it iene type faaks wat mear as by it oare - steane ynlikens en tearens foarop. De ynkoarte nammen dêrfoaroer lykje krekt de flinkens, kordatens en robústens fan de drager fan de namme te beklamjen. Dat komt moai út yn it sitaat út Schurer (1939:20-21) yn (18) ${ }^{26}$ :

25. Yn Grins is der in (frij nije) saak dy't himsels as 'het Toetjesparadijs' affisearret. De namme dêrfan is Toet. Hjir kin ek wurdboarterij mei anneks wêze: it toetje krijt men troch de toet yn it liif.

26. Op side 6 fan de earste jefte fan it blêd De Nije (desimber 2018) stiet in fraachpetear mei Lutz Jacobi (foarsitter fan 'e Waadferiening). De kop boppe it artikel is: "Lutz stoerder as Lutske". Yn it artikel seit frou Jacobi: "Doe't ik in jier of sechtjin, santjin wie, hie ik in freondinne dy’t Wietske hjitte. Op in dei hawwe wy besletten om ússels net mear Wietske en Lutske te neamen, mar Wietz en Lutz, dat fûnen wy stoerder'. De ynkoarte namme wurdt hjir kleardernôch mei de eigenskip/it konsept 'stoer' assosjearre. 
(18) sitaat út Schurer (1939)

Voor Friesche ooren klinken die vrl. namen kort, afgebeten, ferm en stoer, "stûf" zegt de Fries. Ze roepen onwillekeurig historische gestalten op van fiere edelvrouwen, zittende op strijdpaarden, de teugels in de linker-, het zwaard in de rechterhand. Er zijn ook Friesche Kenau's geweest!

En het zijn dezelfde namen, die nog heden ten dage de schoolmeisjes, spontaan als ze zijn in de hitte van het spel, -met verwaarloozing van latere, zachtere vormen elkaar toeroepen: "Do moast, Sjouk! 't Is dyn beurt, Hylk! Né Foek, det 's net earlik en do kreauwst (schelmen) ek mei, smoarge Lutsk!"

Al kinne se út mear as ien wurdlid bestean, ienwurdliddichheid is karakteristyk foar fierwei de measte ynkoarte nammen. Neffens Schurer hearre sokke nammen de Friezen as "kort" en "afgebeten" oan, dêr't er yn ien sike de kwalifikaasjes "ferm en stoer ("stûf")" oan taheakket. Soe it no sa wêze kinne, dat dy ienwurdliddichheid de konnotaasje 'flink', 'kordaat' en 'robúst' opropt? Of moat men dy prinsepeal op rekken skriuwe fan it missen fan de ferlytsingsútgong? Hoe dan ek, der liket ditoangeande in ferskil te wêzen tusken bygelyks Hil en Ibel oan 'e iene en Hiltsje en Ibeltsje oan 'e oare kant. Dat wol lykwols net sizze, dat in ynkoarte namme net in hypokorisme is. Beskiedend liket my hjir ta, dat de ynkoarte namme oars is as de grûnfoarm. Dy ôfwiking jout sa'n namme in net-neutraal karakter en dêrtroch drukt er in beskaat 'affekt' út.

Hypokorismen wurde op ûnderskate wizen brûkt. Ferlytsingsnammen en $y$ nammen hawwe it meast fan gewoane, neutrale persoansnammen en ien dermei oansprekke is dan ek hiel gewoan. Dat is oars mei flaainammen en ynkoarte nammen. Dy kinne skoan as oantsjutting fan spesifike persoanen brûkt wurde, mar ien dermei oansprekke liket mear foarbeholden te wêzen oan minsken dy't de persoan yn kwestje goed kenne. Ynkoarte nammen lykje ek net oan bern jûn te wurden. De konnotaasje fan 'flinkens', 'kordatens' en 'robústens' liket net goed te passen by lytse poppen. Fierders falt it op, dat men ynkoarte nammen minder yn rou-advertinsjes sjocht as de oare hypokorismen. Men koe jin yntinke, dat de neibesteanden de 'flinkens', 'kordatens' en 'robústens' fan de ferstoarne net beklamje, mar it byld fan him/har leaver 'fermyldzje' en 'fersêftsje' wolle. Mar dat is út soarte in frijwat spekulative 'ferklearring'. 


\section{By einsluten}

Yn dit stik haw ik oannimlik meitsje wollen, dat in ferlytsingsnamme in ynkoarte foarm neist him hawwe kin. De formele relaasje tusken dy twa lit him beskriuwe mei it skema 'ynkoarte namme = ferlytsingsnamme sûnder ferlytsingssuffiks'. Ynkoarte nammen nimme de formele en semantyske eigenskippen fan ferlytsingsnammen oer en binne yn dy sin 'parasitêr' fan aard. Dat hat men ek mei $y$-nammen, dy't op flaainammen 'parasitearje' en dêr eigenskippen fan oernimme kinne (lykas by Durk (grûnfoarm) - Dukke (flaainamme) - Dukky (y-namme)).

As wy de ynkoarte nammen ynpasse yn it oersjoch yn (4) hjirboppe, dan kin dat útwreide wurde ta (19):

$$
\begin{aligned}
& \text { oerienkomsten en ferskillen tusken ferlytsingsnammen, flaainammen, } \\
& \text { y-nammen en ynkoarte nammen }
\end{aligned}
$$

$\begin{array}{lllll} & \text { ferl.namme } & y \text {-namme } & \text { flaainamme } & \text { ynk. namme } \\ \text { man \& frou } & + & + & + & + \\ \text { 'rekursiviteit' } & + & - & + & - \\ \text { suffiks } & + & + & - & - \\ \text { trogee } & +/- & + & + & - \\ \text { tal wurdlidden } & \geq 2 & 2 & \leq 2 & 1(2)\end{array}$

Oan (19) kin ôfnommen wurde, dat ynkoarte nammen in eigen plak yn it lânskip fan de Fryske hypokorismen hawwe.
Fryske Akademy
Rijksuniversiteit Groningen
Ljouwert
Frysk Ynstitút

\section{LITERATUER}

Hoekema, T. (1986)

'Brekking en lûdferkoarting yn eigennammen', Us Wurk 35, pp. 55-56.

Hoekstra, J. (1998a)

Fryske wurdfoarming, Ljouwert, Fryske Akademy.

Hoekstra, J. (1998b)

'Iampk ist en iette iamcker wirtet mecke: Jitris oer it komôf fan Nijfrysk jamk', It Beaken 60, pp. 1-14. 
Nübling, D. (2017)

'The growing distance between proper names and common nouns in German: On the way to onymic schema constancy', Folia Linguistica 51, pp. 341-367.

Schurer, B.J. (1939)

De Friesche eigennamen verklaard door B.J. Schurer, Populair-wetenschappelijk voor het Friesche en Nederlandsche Volk beide. Merkwaardige Afkomst Vorming Beteekenis. SLEUTEL voor de VERKLARING van het grootste deel der NEDERLANDSCHE FAMILIE- EN PLAATSNA$M E N$. Samenhang met taalwording? Uitgave van den schrijver, Den Dolder 1939.

Visser, W. (2002)

'Brekking as ferkoarting: in diagroane analyze', It Beaken 64, pp. 187246.

Visser, W. (2003)

'Patroanen yn ferlytsingsnammen', yn: P. Boersma e.o. (red.), Philologia Frisica anno 2002, Ljouwert, Fryske Akademy, pp. 263-305.

Visser, W. (2010)

'Flaainammen yn it Frysk', Us Wurk 59, pp. 1-78.

Visser, W. (2016)

' $y$-nammen yn it Frysk', Us Wurk 65, pp. 91-145. 